\title{
GaSb-based integrated lasers and photodetectors on a Silicon-On-Insulator waveguide circuit for sensing applications in the shortwave infrared
}

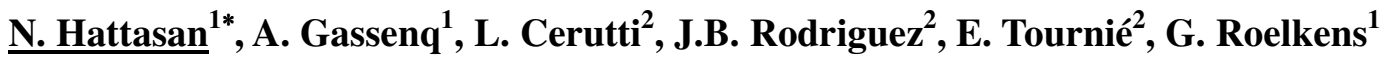 \\ ${ }^{1}$ Photonics Research Group-UGent/imec, Sint-Pietersnieuwstraat 41, 9000 Gent, Belgium \\ ${ }^{2}$ Université Montpellier 2 - CNRS, UMR 5214, Place Bataillon, 34095 Montpellier, France
}

The interest in sensing liquids and gases such as $\mathrm{CH}_{4}$ and $\mathrm{CO}$ in the shortwave infrared has increased over the past decade due to the low water absorption in this region. Conventional spectroscopic techniques, which may offer high accuracy, require expensive and bulky sources and detectors. However, a compact, low-power system is strongly desirable for many applications.

$\mathrm{GaSb}$ and its compounds are an excellent semiconductor material for the shortwave infrared. Several room temperature operating lasers and detectors have thus far been demonstrated using GaSb, making it suitable for integrated active components [1]. SiliconOn-Insulator (SOI), on the other hand, offers mass production of optical circuits with CMOS compatibility. This makes it ideal as a passive integrated optical circuit platform. Combining SOI and GaSb optoelectronic components could thus yield a compact, efficient spectroscopic detection system.

Here, we report integrated InGaAsSb (grown lattice-matched on a GaSb substrate) photodetectors on SOI and integrated Fabry-Perot (FP) lasers. The epitaxial layers are transferred onto the $\mathrm{Si}$ waveguide circuit by a die-to-wafer bonding process using Benzocyclobutene (BCB) as the bonding agent. The substrate is completely removed before device processing. For photodetectors, we study two light coupling techniques from the SOI waveguide circuit to the integrated photodetector: evanescent coupling and grating-assisted coupling. High responsivity $(>1 \mathrm{~A} / \mathrm{W})$ of the evanescent photodiode and $0.4 \mathrm{~A} / \mathrm{W}$ for gratingassisted coupling at $2.2 \mu \mathrm{m}$ is achieved. The dark current is $\sim 4 \mu \mathrm{A}$ at $-1 \mathrm{~V}$. SEM images of both designs are shown in Fig.1a and 1b.

We also demonstrate thin-film GaSb-based FP lasers integrated on a carrier substrate. The $15 \mu \mathrm{m}$ x $400 \mu \mathrm{m}$ device operates at $\sim 2.02 \mu \mathrm{m}$ wavelength in continuous wave at room temperature. A threshold current of $\sim 31 \mathrm{~mA}$ in pulse regime and $49.7 \mathrm{~mA}$ in continuous wave are achieved. The integrated laser operates up to $35^{\circ} \mathrm{C}$. Figure $1 \mathrm{c}$ represents its spectrum at different bias current.
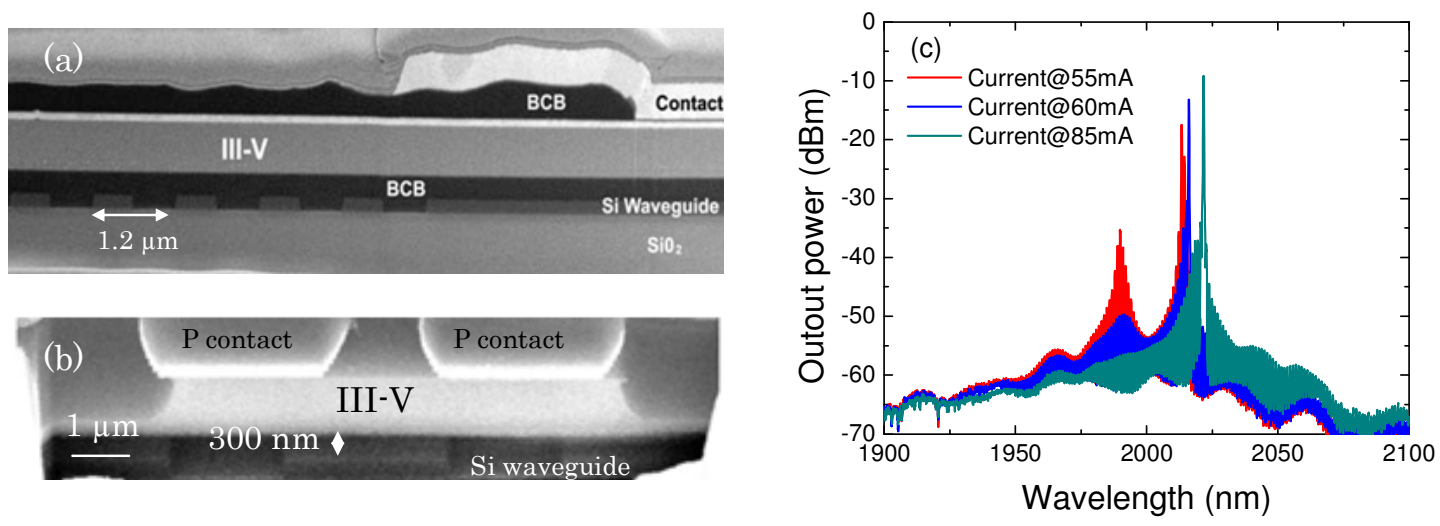

Fig.1 SEM image of the realized components (a) Grating-assisted coupling, ( b) Evanescent coupling , (c) Spectrum at different bias current of the realized laser

[1] A. Bauer, K. Rößner, T. Lehnhardt, M. Kamp, S. Höfling, L. Worschech and A. Forchel, Semicond. Sci. Technol. 20, 014032, 2011

* Corresponding author: email: nannicha.hattasan@intec.ugent.be 
150 words:

We report integrated InGaAsSb (grown lattice-matched on a GaSb substrate) photodetectors on SOI and integrated Fabry-Perot (FP) lasers. The epitaxial layers are transferred onto the $\mathrm{Si}$ waveguide circuit by a die-to-wafer bonding process using Benzocyclobutene (BCB) as the bonding agent. The substrate is completely removed before device processing. For photodetectors, we study two light coupling techniques from the SOI waveguide circuit to the integrated photodetector: evanescent coupling and grating-assisted oupling. High responsivity $(>1 \mathrm{~A} / \mathrm{W})$ of the evanescent photodiode and $0.4 \mathrm{~A} / \mathrm{W}$ for gratingassisted coupling at $2.2 \mu \mathrm{m}$ is achieved. The dark current is $\sim 4 \mu \mathrm{A}$ at $-1 \mathrm{~V}$.

We also demonstrate thin-film GaSb-based FP lasers integrated on a carrier substrate. The 15 $\mu \mathrm{m} \times 400 \mu \mathrm{m}$ device operates at $\sim 2.02 \mu \mathrm{m}$ wavelength in continuous wave at room temperature. A threshold current of $\sim 31 \mathrm{~mA}$ in pulse regime and $49.7 \mathrm{~mA}$ in continuous wave are achieved. The integrated laser operates up to $35^{\circ} \mathrm{C}$. 